\title{
An Adolescent Presented With Hemoptysis: Pulmonary Artery Aneurysm in Pediatric Behçet's Disease
}

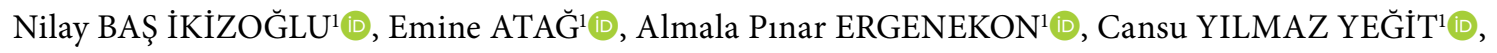

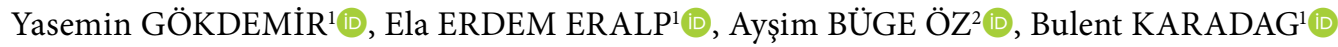 \\ ${ }^{1}$ Department of Pediatrics, Division of Pediatric Pulmonology, Marmara University School of Medicine, Istanbul, Turkey \\ ${ }^{2}$ Department of Pathology, Istanbul University Cerrahpaşa Faculty of Medicine, Istanbul, Turkey
}

\begin{abstract}
Behçet's disease (BD) is a rare autoimmune and chronic inflammatory vasculitis, characterized by relapsing episodes of oral aphthous and genital ulcers, skin lesions, ocular lesions and vascular involvement. Pulmonary artery involvement is rare in BD but it carries a high mortality risk. In this article, we report a 15-year-old male patient presented with a two-month history of hemoptysis, cough, fewer and weight loss. On physical examination, auscultation revealed decreased breath sounds at left lung base. Bronchoscopy showed narrowed left lower lobe bronchus due to the external compression. Computed tomography angiography revealed multiple bilateral pulmonary artery aneurysms. Pathergy test was positive and he was diagnosed with BD. BD should be considered in the differential diagnosis of childhood hemoptysis.

Keywords: Connective tissue disease, hemoptysis, pediatrics, pulmonary.
\end{abstract}

Behçet's disease (BD) is a rare disease, characterized by chronic perivascular inflammation, recurrent oral and genital ulcers, ocular inflammation and multisystemic involvement due to vasculitis. It is more common in countries along the old Silk Road such as Turkey, Greece, and Israel. The etiology is unknown, while epidemiologic data point to the role of both genetic and environmental factors. ${ }^{1,2}$ BD usually presents at third to fourth decades and is rare in children. Vasculitis in BD can affect both arteries and veins; however, venous involvement is more common. Although the most common arterial involvement is pulmonary artery aneurysm (PAA), it occurs very rarely in $\mathrm{BD}$. In a retrospective analysis, the prevalence of pulmonary artery involvement including PAA and pulmonary artery thrombosis was found to be
$2 \% .^{3}$ To our knowledge, there is no data about the prevalence of PAA in children with $\mathrm{BD}$. Patients with PAA usually present with hemoptysis and carry a high risk of mortality. ${ }^{4}$ It should be kept in mind that some life-threatening features in the pediatric population may occur earlier than mucocutaneous findings.

In this article, we report an adolescent diagnosed with $\mathrm{BD}$ to emphasize the importance of vascular etiologies such as BD in the differential diagnosis of hemoptysis even in the pediatric age group.

\section{CASE REPORT}

A 15-year-old male patient presented with productive cough, hemoptysis, fever, and weight

Received: February 27, 2019 Accepted: June 24, 2019 Published online: October 16, 2019

Correspondence: Emine Atağ, MD. Marmara Üniversitesi Pendik Eğitim ve Araştırma Hastanesi, Çocuk Pulmonoloji Bilim Dalı, 34899 Pendik, İstanbul, Turkey. Tel: +90533-6850087 e-mail:emineatag@gmail.com 
loss. His symptoms had started two months before. Hemoptysis resolved after a course of co-amoxiclav with the diagnosis of pneumonia, but intermittent productive cough and fever persisted over the two months, while he lost $4 \mathrm{~kg}$ within this period. On past medical history, he had recurrent oral and genital ulcers over the last year. Family and natal history was not remarkable. A written informed consent was obtained from the legal guardians of the patient.

On physical examination; his general condition was fair, he had fever (axillary: $38.5^{\circ} \mathrm{C}$ ) and seemed fatigued. His height and weight were within the normal range. He had decreased breath sounds at the left lung base. Oxygen saturation was 98\% on room air. He had digital clubbing, and other system examinations were normal.

Laboratory results were as follows: hemoglobin: $12.6 \mathrm{~g} / \mathrm{dL}$, white blood cell: $16,000 / \mathrm{mm}^{3}$, platelet: $323,000 / \mathrm{mm}^{3}$, erythrocyte sedimentation rate: $128 \mathrm{~mm} /$ hour, C-reactive protein: $218 \mathrm{mg} / \mathrm{L}$. He had no atypical cells on the blood smear. Capillary gas, urine, coagulation, and biochemical analysis were normal.

Chest X-ray showed retrocardiac hyperdensity on admission (Figure 1). Diagnostic investigations for tuberculosis were all negative. On bronchoscopy; left lower lobe bronchus was narrowed due to the external compression and abundant viscous secretions were observed.

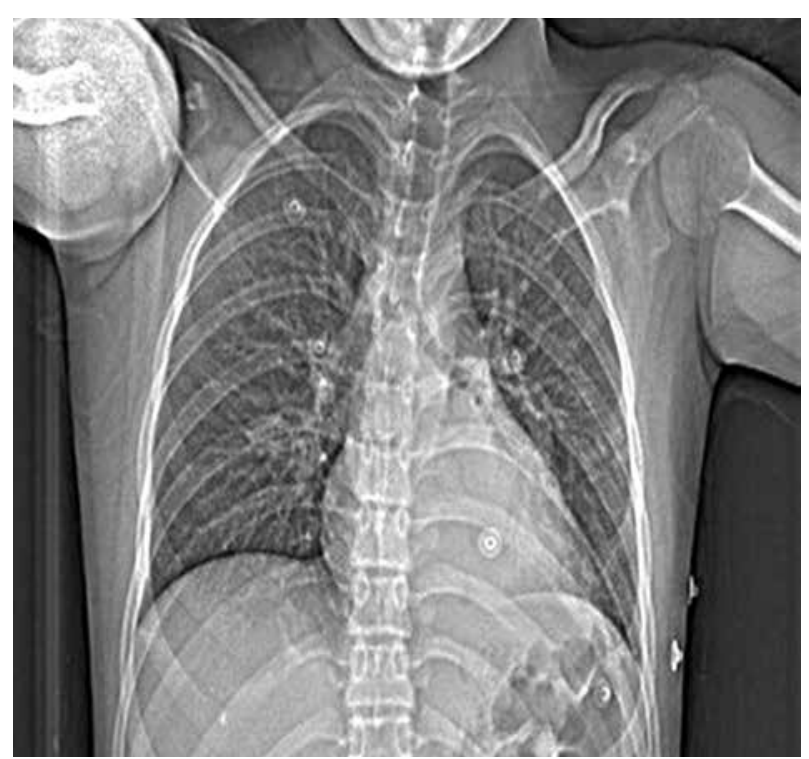

Figure 1. Chest X-ray demonstrates retrocardiac opacity.
Computed tomography (CT) angiography was performed, which was consistent with multiple PAAs bilaterally (Figure 2). Diagnostic investigations such as echocardiogram and lower extremity venous Doppler ultrasonography, renal function evaluation, eye examination, abdominal ultrasound, and rheumatologic tests revealed no pathological findings. Our patient's investigational tests for PAA were negative for cardiovascular disease, pulmonary hypertension or any infection.

Pathergy test was performed due to the presence of recurrent oral and genital ulcers, supporting the diagnosis of BD. Pathergy test was positive and he was diagnosed as BD according to the history, physical examination and laboratory findings. Monthly intravenous cyclophosphamide pulses combined with high dose methyl-prednisone were initiated and followed by prednisolone $2 \mathrm{mg} / \mathrm{kg} /$ day. Afterwards, left lower lobectomy was performed. Pathological examination revealed PAA with thrombus in the lumen, fibrosis and lymphoplasmacytic inflammatory cell infiltration adjacent to small- and mediumsized vessels (Figure 3). He was clinically well after lobectomy and admitted for the second pulse immunosuppressive therapy. Meanwhile, CT angiography was performed to evaluate the aneurysm at the right lower lobe which showed regression of PAA from $16 \mathrm{~mm}$ to $6 \mathrm{~mm}$ in diameter. Endovascular embolization was

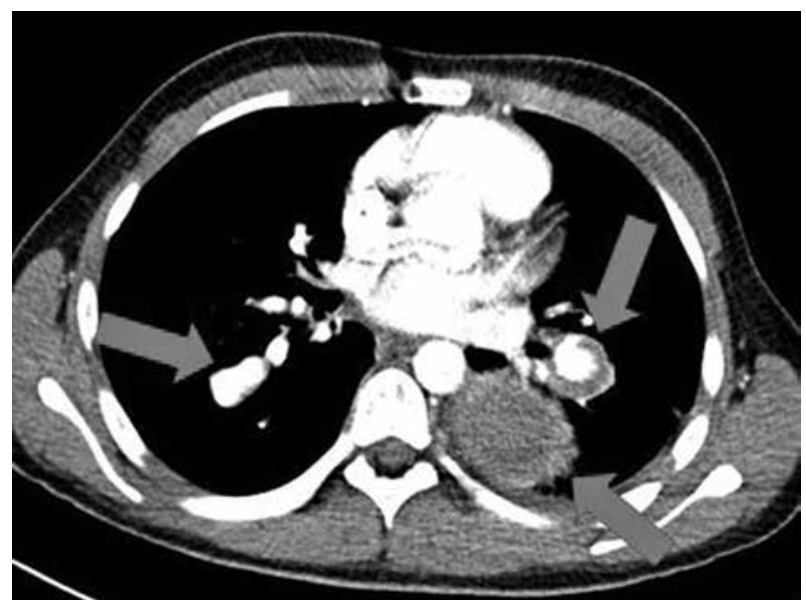

Figure 2. Computed tomography angiography was consistent with multiple pulmonary artery aneurysms bilaterally. Arrow on right lung shows a totally thrombosed aneurysm. Arrows on left side show partially thrombosed aneurysms. 


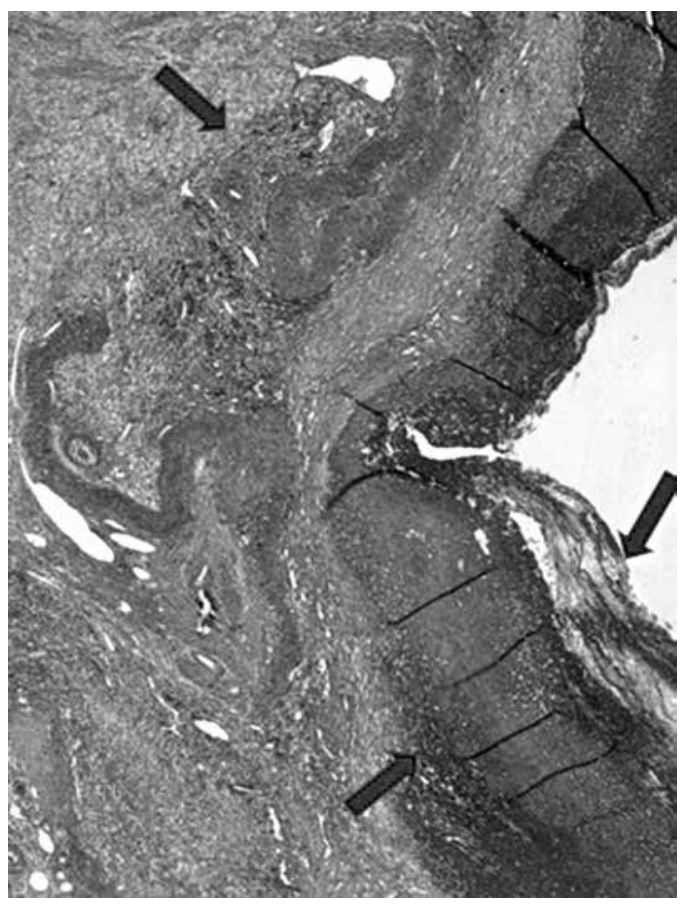

Figure 3. Pathological examination: two arrows at bottom show pulmonary artery aneurysm with thrombus in lumen, while arrow at top shows fibrosis and inflammatory cell infiltration adjacent to a small-sized vessel $(\mathrm{H}-\mathrm{E} \times 40)$.

planned for the right lower lobe PAA. However, unfortunately, he presented with massive hemoptysis five days later. The patient passed away with massive hemoptysis originating from the right lower lobe PAA.

\section{DISCUSSION}

Behçet's disease is characterized by chronic perivascular inflammation. It is more common in the male sex. Male sex and early onset disease are associated with poor prognosis. The main manifestations of $\mathrm{BD}$ are oral and genital ulcers. BD may affect many other organs such as eyes, skin, and joints. ${ }^{1,2}$ Major vessel involvement occurs in 20 to $30 \%$ of $\mathrm{BD}$. It mainly affects venous vessels and presents with thrombosis.

Pulmonary manifestations including vascular and parenchymal involvement occur in 1 to $7 \%$ of all cases. Pulmonary complications develop mainly in the first few years of the onset of the disease and have male predominance. There is no specific distribution in terms of age; a retrospective analysis of $\mathrm{BD}$ patients showed that the mean age was similar in patients with and without pulmonary involvement. ${ }^{5}$ Pulmonary vascular involvement may be arterial or venous. $\mathrm{PAA}$ is rare in $\mathrm{BD}$; however, PAA is the most typical manifestation of pulmonary artery complications of $\mathrm{BD}$. Hemoptysis is the most common and typical symptom, followed by dyspnea and cough. Patients with PAA usually present with massive hemoptysis, PAAs with a diameter $>3 \mathrm{~cm}$ are associated with a high risk of mortality. ${ }^{3}$ On the other hand, the most common causes of hemoptysis in childhood are infections. In a study examining the causes of hemoptysis in children, the frequency of vascular anomalies was only $6 \% .^{6}$ Although vascular anomalies rarely cause hemoptysis, they should be considered in the differential diagnosis as they can cause life-threatening bleeding. PAA is usually located bilaterally in branches of the lower lobes. ${ }^{1,2,7-9}$ Our patient also had bilateral PAA. This finding was consistent with the results of a study reporting $47 \mathrm{BD}$ patients with pulmonary artery involvement, which revealed bilateral PAA with a rate of $57 \%$ in patients. ${ }^{10}$ PAA is a significant cause of mortality in $\mathrm{BD}$; despite treatment, unfortunately, the mortality rate is $25 \% .^{3}$

Diagnosis depends on clinical findings. International Society for BD proposed criteria for diagnosing $\mathrm{BD}$. These criteria require the presence of recurrent oral ulcers plus two of the following symptoms in the absence of other systemic diseases: recurrent genital ulcerations, eye lesion, skin lesions (erythema nodosum, papulopustular lesions, or acneiform nodules), or a positive pathergy test. Our patient had recurrent oral and genital ulcers and positive pathergy test fulfilling the diagnostic criteria., ${ }^{1,7,11}$ If the symptoms start before the age of 16 , it is defined as pediatric $\mathrm{BD}$. According to the diagnostic criteria for pediatric $\mathrm{BD}$, individuals who have three of six items (recurrent oral and genital ulceration, skin, ocular, neurological or vascular involvement) are defined to have pediatric $\mathrm{BD} .^{7}$ Our patient's symptoms started before the age of 16 and he had three symptoms (oral and genital ulceration and vascular involvement) that confirmed the pediatric $\mathrm{BD}$ as well.

Symptom control and suppression of inflammation are the mainstays of the 
management. Interventional treatments such as embolization and lobectomy may have been necessary for patients refractory to medical treatments. ${ }^{12,13}$ PAA in the left lower lobe of our patient was huge, thus lobectomy was the best therapy option, and it was performed after cyclophosphamide pulse therapy. Although the size of PAA in the right lower lobe reduced to $6 \mathrm{~mm}$, the patient had a massive hemoptysis attack before endovascular embolization. Bilateral lower lobectomy may be a better treatment option in our case with giant PAAs. In a study on nine patients who underwent surgical treatment, Tuzun et al. ${ }^{14}$ concluded that four out of six patients who underwent lobectomy were still alive and that lobectomy was an acceptable method in patients who did not respond to medical treatment.

Consequently, there are only a few childhood $\mathrm{BD}$ case reports presented with PAA in the literature..$^{15-18}$ This case highlights the importance of considering $\mathrm{BD}$ in pediatric patients with hemoptysis. BD with PAA is associated with a high risk of morbidity and mortality and should be treated promptly.

\section{Declaration of conflicting interests}

The authors declared no conflicts of interest with respect to the authorship and/or publication of this article.

\section{Funding}

The authors received no financial support for the research and/or authorship of this article.

\section{REFERENCES}

1. Hatemi G, Yazici Y, Yazici H. Behçet's syndrome. Rheum Dis Clin North Am 2013;39:245-61.

2. Saadoun D, Wechsler B. Behçet's disease. Orphanet J Rare Dis 2012;7:20.

3. Seyahi E, Yazici H. Behçet's syndrome: pulmonary vascular disease. Curr Opin Rheumatol 2015;27:18-23.

4. Seyahi E. Behçet's disease: How to diagnose and treat vascular involvement. Best Pract Res Clin Rheumatol 2016;30:279-95.

5. Edrees A, Naguib S, El Menyawi M, Ismail I, Nagah $\mathrm{H}$.
Pulmonary manifestations in a group of patients with Behcet's disease. Int J Rheum Dis 2017;20:269-75.

6. Vaiman M, Klin B, Rosenfeld N, Abu-Kishk I. Pediatric pulmonary hemorrhage vs. extrapulmonary bleeding in the differential diagnosis of hemoptysis. Cent Asian J Glob Health 2017;6:284.

7. Koné-Paut I, Shahram F, Darce-Bello M, Cantarini L, Cimaz R, Gattorno M, et al. Consensus classification criteria for paediatric Behçet's disease from a prospective observational cohort: PEDBD. Ann Rheum Dis 2016;75:958-64.

8. Celik S, Yazici Y, Sut N, Yazici H. Pulmonary artery aneurysms in Behçet's syndrome: a review of the literature with emphasis on geographical differences. Clin Exp Rheumatol 2015;33:S54-9.

9. Calamia KT, Schirmer M, Melikoglu M. Major vessel involvement in Behçet's disease: an update. Curr Opin Rheumatol 2011;23:24-31.

10. Seyahi E, Melikoglu M, Akman C, Hamuryudan $\mathrm{V}$, Ozer $\mathrm{H}$, Hatemi $\mathrm{G}$, et al. Pulmonary artery involvement and associated lung disease in Behçet disease: a series of 47 patients. Medicine (Baltimore) 2012;91:35-48.

11. Criteria for diagnosis of Behçet's disease. International Study Group for Behçet's Disease. Lancet 1990;335:1078-80.

12. Emmi G, Bettiol A, Silvestri E, Di Scala G, Becatti M, Fiorillo C, et al. Vascular Behçet's syndrome: an update. Intern Emerg Med 2018 Nov 29. [Epub ahead of print]

13. Marshall SE. Behçet's disease. Best Pract Res Clin Rheumatol 2004;18:291-311.

14. Tuzun H, Seyahi E, Guzelant G, Oz B, Batur S, Demirhan $\mathrm{O}$, et al. Surgical treatment of pulmonary complications in Behçet's syndrome. Semin Thorac Cardiovasc Surg 2018;30:369-78.

15. Lee WY, Hoon CS, Kim HR. Massive hemoptysis caused by atypical Behcet's disease. Korean J Thorac Cardiovasc Surg 2014;47:178-80.

16. Agha A, Bella AM, Assiri AH, Al-Hakami M. Can Behcet's disease related pulmonary arterial aneurysms be completely resolved? Open Rheumatol J 2011;5:88-90.

17. Takahama M, Yamamoto $\mathrm{R}$, Nakajima $\mathrm{R}$, Tada $\mathrm{H}$. Successful surgical treatment of pulmonary artery aneurysm in Behçet's syndrome. Interact Cardiovasc Thorac Surg 2009;8:390-2.

18. Alibaz-Oner F, Karadeniz A, Ylmaz S, Balkarl A, Kimyon G, Yazc A, et al. Behçet disease with vascular involvement: effects of different therapeutic regimens on the incidence of new relapses. Medicine (Baltimore) 2015;94:e494. 